

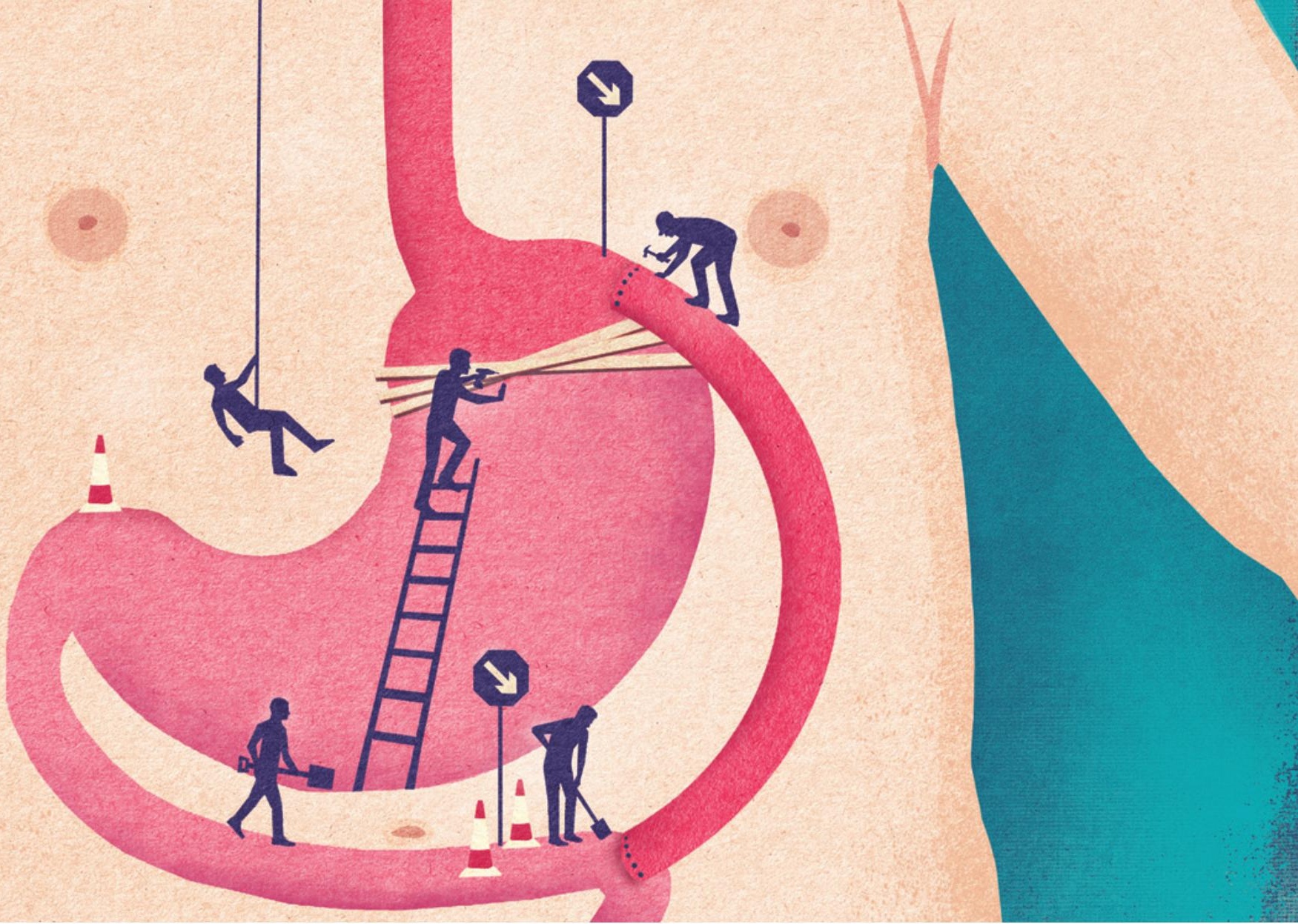

was mechanical, patients would simply eat lots of small meals. "That got the field wondering, what's going on with hunger, here?"

In 2002, Cummings and his colleagues identified one of the first biochemical markers associated with the bypass. They had tracked blood levels of ghrelin, the 'hunger hormone' produced by cells in the gastrointestinal tract, in more than two dozen people. Normally, ghrelin levels rise sharply when the stomach is empty and then drop after a meal. Surgery suppressed these fluctuations, Cummings found ${ }^{4}$. The normal peaks and valleys of ghrelin production went pancake flat. "It's pretty dramatic," he says.

But getting a better handle on the mechanisms required an animal model. Lee Kaplan, director of the Massachusetts General Hospital Weight Center in Boston, looked to rats - a daunting task given their tiny innards. He recruited a young surgeon from Greece, Nicholas Stylopoulos, and the duo, along with a few other research groups, began to publish papers on what happened to the animals after surgery. The research has shown that just like in people, bypass surgery stabilizes glucose lev$\mathrm{els}^{5}$, boosts metabolism ${ }^{6}$ and steers the animals to choose low-fat over high-fat meals ${ }^{7}$.

\section{GUT MICROBES}

A potential explanation could lie in the trillions of microbes that reside in the gut. In 2009, Rosa Krajmalnik-Brown from Arizona State University in Tempe and her colleagues sequenced the bacterial genes present in faeces from three people who had received a gastric bypass. Compared with obese and normal-weight controls, their guts contained proportionally fewer bacteria from the usually abundant Firmicutes phylum, and excess levels of the Gammaproteobacteria class ${ }^{8}$."Even with that small sample size we were able to get statistically significant differences because the microbiota changed so drastically," Krajmalnik-Brown says.

The researchers do not know why these particular changes occurred, but they say it could be because Firmicutes die when oxygen is present, and shortening the gastrointestinal tract means that oxygen that is normally consumed in the small intestine reaches the colon. Alternatively, the changes could occur because food is being digested faster. (The group did not test microbial make-up in individuals before surgery, but is now working on a follow-up study that compares before and after.) A similar shift in gut flora has been reported in rats undergoing a gastric bypass ${ }^{9}$.

Whether this bacterial shift drives a change in health is hard to say, but there are some indications that the microbes contribute to metabolic changes. Kaplan and his colleagues performed a gastric bypass on obese mice, then transplant the altered gut bacteria into mice bred to be microbe-free. These recipient mice were not obese, but still lost about $5 \%$ of their weight after the transplant ${ }^{10}$ (see Nature http://doi.org/tjq; 2013).

This research and other strands of evidence suggest that metabolic regulation could begin in the gut, which has the ability to send messages to the brain, liver, pancreas, kidneys and immune system. "The idea that a lot of the information starts at the gut is a relatively new concept," says Kaplan.

For example, researchers have now found that bile acids have a role in signalling. These fluids help to emulsify fats so that the lipids are metabolized more efficiently, but they also act as hormones, signalling to receptors in the gut. Randy Seeley, a neuroscientist at the University of Michigan Health System in Ann Arbor, and his colleagues decided to look at what happens when one of these bile-activated-receptors - the farsenoid-X receptor (FXR), which helps to regulate glucose metabolism - is deleted in mice.

The researchers overfed both mutant and control mice until they were fat, and then did a vertical sleeve gastrectomy. (This procedure shrinks the stomach like a gastric bypass does, but does not circumvent any of the small intestine.) A week after surgery, both types of mice lost a lot of weight. By the fifth week, however, only the control mice had managed to keep it off; the mutants had gained it all back ${ }^{11}$. Without FXR and the messages carried by bile acids, the surgery fails to work

Intriguingly, the control mice, but not the mutants, showed a notable increase in the abundance of Roseburia, a Firmicutes bacterium that tends to be suppressed in people 
with diabetes, suggesting that FXR and its related biological pathways could turn out to be therapeutic targets in this disease.

Bile-acid and bacterial changes could affect the gut's communication with organs responsible for the glucose dysregulation that causes diabetes. But a study published last year ${ }^{12}$ suggests that the gut itself shows changes in glucose metabolism after surgery (see Nature http://doi.org/tjr; 2013).

Using a rat model of gastric bypass, Stylopoulos, who now runs his own laboratory at Boston Children's Hospital, and his colleagues showed that the 'Roux limb' - the piece of intestine that runs from the stomach pouch to the reconnected intestine - expands dramatically in width and length after surgery. "It really doubles in size," Stylopoulos says, and it stays that way. That makes sense, because without a full-sized stomach, the tissue must adapt to heaps of undigested food. But the limb's rapid growth requires a lot of energy, which comes from glucose. The changing organ starts to use more glucose, and the change is maintained over time, Stylopoulos says. "Essentially, the intestine becomes a bigger and a more hungry organ that needs more glucose than before."

Stylopoulos believes that this tissue growth in the gut is the main driver of the surgery's remarkable metabolic benefits - not the reduction in calorie intake. "Surgery works because it changes the physiology," he says.

Weight loss is still important, however, because it triggers a series of changes that help to curb diabetes.

\section{PROBLEMS IN TRANSLATION}

How well do these findings translate into people? "These are elegant studies," says Samuel Klein, director of the Center for Human Nutrition at Washington University School of Medicine in St Louis, Missouri. But, he asks: "Is the bariatric surgical procedure in a rodent the same as in a human?"

Klein allows that just like rodents, people have a marked improvement in bloodglucose regulation within days of bypass surgery. But that could be because their caloric intake goes from around 4,000 calories a day to just 400, he says. "Anyone who has abdominal surgery is not going to be very hungry after the operation."

Rates of diabetes remission are much higher after gastric bypass than after gastric banding — in which a silicone band squeezes around the stomach to restrict the flow of food (see 'Surgical selection'). Animal studies suggest that that is because the bypass alters metabolism in a way that banding does not, but Klein believes that it is simply because people who have a bypass tend to lose much more weight.

To probe this, Klein compared people who had lost one-fifth of their weight after gastric bypass with those who lost the same

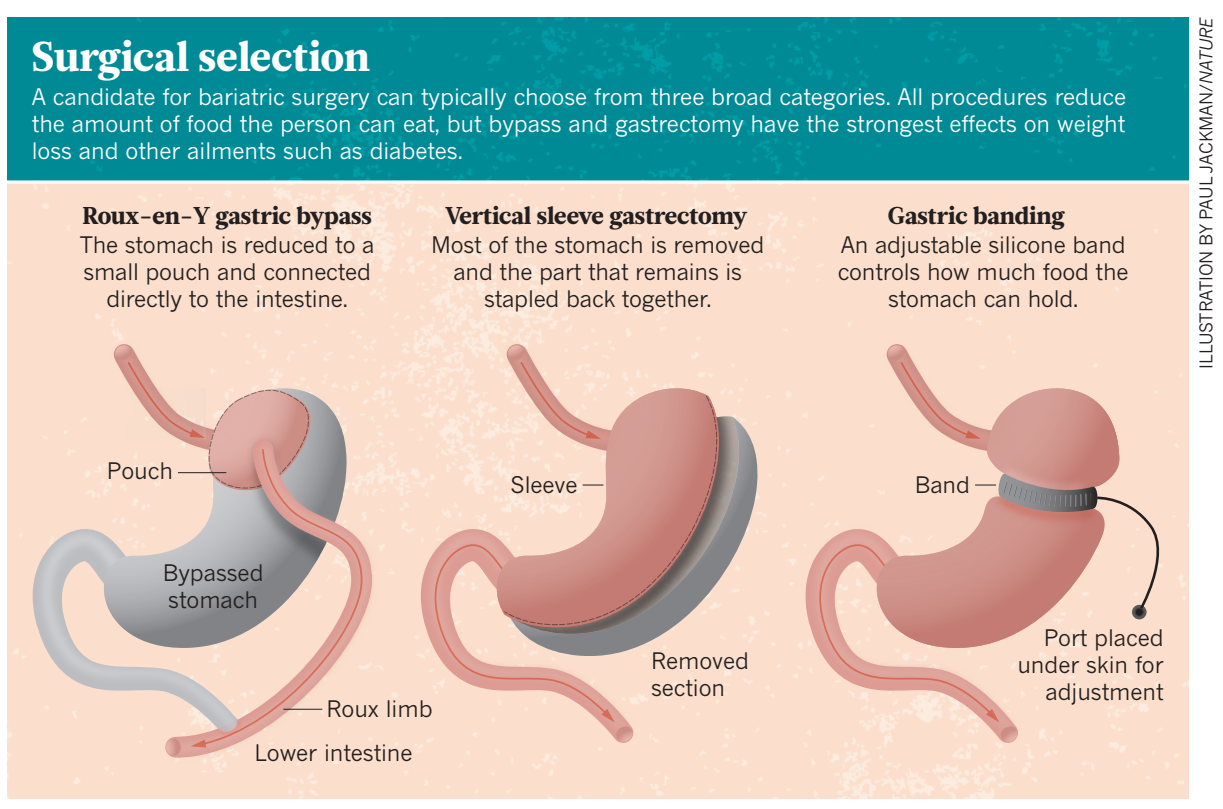

amount with banding. All patients showed dramatic improvements in glucose tolerance, insulin sensitivity and the function of pancreatic $\beta$-cells, which release insulin ${ }^{13}$. "We did not see any hint" of differences between the groups, he says. The major caveat of this study is that none of the volunteers had diabetes, so Klein's group is now repeating the study in people with the disease. "It could be a whole different ball game," he says.

Still, he agrees that rodent studies provide a relatively quick way to investigate specific biological pathways and test hypotheses about why only some procedures curb diabetes and why certain patients are more likely to benefit than others. By testing individual pathways, researchers hope that they can develop personalized treatments - whether drugs, probiotics or lifestyle changes - that change the specific pathway that has gone awry in a patient.

For Courcoulas, the variability and unpredictability in patient response - in both weight loss and diabetes remission - is the most important issue that animal studies could address. When talking to prospective patients about their surgical options, she frequently refers to a study she published last year $^{14}$ that tracked nearly 2,500 people who had undergone various types of bariatric surgery.

After three years, those who received gastric banding had lost, on average, about $16 \%$ of their weight, whereas those who had a gastric bypass lost $32 \%$. Banding also led to partial remission of diabetes in $29 \%$ of people, compared with $68 \%$ for bypass. In general, Corcoulas notes, most people lose a lot of weight in the first six months, irrespective of the procedure. But after that, they diverge wildly: some people continue to lose at a rapid clip, others plateau and still others gain some back. This uncertainty partly explains why so few people who are eligible for surgery choose to have it, she says. At her centre, nearly 1,500 people a year attend group informational sessions to learn the basics of weight-loss surgery. Only 1,000 of them will elect to talk to a surgeon, and 700 will go on to have an operation.

"The big question is, what are the factors, the predictors for someone's success after surgery?" Courcoulas says. Clinical studies have identified some contributors - iron deficiency, liver fibrosis and being older than 50 years, for instance, are all associated with less weight loss ${ }^{15}$. But none of these is absolute. The only thing clear, Courcoulas says, is the need to identify better biological markers. "My colleagues in basic science," she says, "are going to be making a big contribution in doing that."

Virginia Hughes is a science journalist based in New York.

1. Adams, T. D. et al. J. Am. Med. Assoc. 308, 1122-1131 (2012).

2. Henrikson, V. Nordisk Medicin 47, 744 (1952). (republished in Obes. Surg. 4, 54-55; 1994.)

3. Pories, W. J., Caro, J. F., Flickinger, E. G., Meelheim, H. D. \& Swanson, M. S. Ann. Surg. 206, 316-323 (1987)

4. Cummings, D. E. et al. N. Engl. J. Med. 346, 1623-1630 (2002).

5. Rubino, F. \& Marescaux, J. Ann. Surg. 239, 1-11 (2004).

6. Stylopoulos, N., Hoppin, A. G. \& Kaplan, L. M. Obesity 17, 1839-1847 (2009).

7. Zheng, H. et al. Am. J. Physiol. 297, R1273-R1282 (2009).

8. Zhang, H. et al. Proc. Natl Acad. Sci. USA 106, 2365-2370 (2009).

9. Li, J. V. et al. Gut 60, 1214-1223 (2011).

10.Liou, A. P. et al. Sci. Transl. Med. 5, 178ra41 (2013).

11.Ryan, K. K. et al. Nature 509, 183-188 (2014).

13. Bradley, D. et al. J. Clin. Invest. 122, 4667-4674 (2012). 2416-2425 (2013).

15.Still, C. D. et al. Obesity 22, 888-894 (2014).
12. Saeidi, N. et al. Science 341, 406-410 (2013).

14. Courcoulas, A. P. et al. J. Am. Med. Assoc. 310, 